\title{
TWISTED SUMS OF BANACH AND NUCLEAR SPACES ${ }^{1}$
}

\author{
PAWEK DOMAŃSKI
}

\begin{abstract}
A twisted sum of (topological vector) spaces $Y$ and $Z$ is a space $X$ with a subspace $Y_{1}$ isomorphic to $Y$ for which $X / Y_{1}$ is isomorphic to $Z$. It splits if $Y_{1}$ is complemented. It is proved that every twisted sum of a Banach space $Y$ and a nuclear space $Z$ splits. Köthe sequence spaces $Z$ for which this holds are characterized. Every locally convex twisted sum of a nuclear Fréchet space $Y$ and a Banach space $Z$ splits too. If $Z$ is superreflexive, then the local convexity assumption on the twisted sum may be omitted. Other results of this kind on Köthe sequence spaces are obtained.
\end{abstract}

Introduction. In this paper topological vector spaces (tvs) and locally convex spaces (lcs) are generally not assumed to be Hausdorff. We define precisely twisted sums as follows: a diagram of tvs and continuous, relatively open linear maps,

$$
0 \rightarrow Y \stackrel{j}{\rightarrow} X \stackrel{q}{\rightarrow} Z \rightarrow 0
$$

is the twisted sum of $Y$ and $Z$ if it is a short exact sequence, i.e., for every map its image is equal to the kernel of the next map. (*) splits if $j(Y)$ is complemented in $X$ or, equivalently, if there is a continuous linear section $s: Z \rightarrow X$ for $q$ (i.e., $q \circ s=$ id). Given a tvs (lcs) $Y$, we say that a tvs (lcs) $Z$ is in the class $S(Y)$ (resp., $\left.S_{c}(Y)\right)$ if every (resp., every locally convex) twisted sum of $Y$ and $Z$ splits [1]. A tvs $Z$ is called a $\mathscr{K}$-space [6] if $Z \in S(Y)$, where $\operatorname{dim} Y=1$.

In $\S 1$ we give some general results concerning twisted sums and their splitting; these are mainly permanence properties for the classes $S(Y)$ and $S_{c}(Y)$. We then apply these results to Köthe sequence spaces $(\$ 2)$ and to nuclear lcs $(\S 3)$; three of the most interesting results are presented below.

N. J. Kalton [5, Theorem 10.3] characterized metrizable Köthe sequence $\mathscr{K}$-spaces. Our Corollary 2.4 shows that, for any nonzero Banach space $Y$, essentially the same characterization is valid for the general Köthe sequence spaces belonging to $S(Y)$, and it is independent of $Y$.

In Theorem 3.3 we prove that whenever $Z$ is a nuclear space and $Y$ a Banach space, then $Z \in S(Y)$. The previously known particular cases of this result used the

Received by the editors October 10, 1984 and, in revised form, April 8, 1985.

1980 Mathematics Subject Classification. Primary 46A12, 46A22, 46A45, 46A99, 46B99; Secondary 46A05, 46A06.

Key words and phrases. Twisted sum, nuclear space, Fréchet space, Banach space, complemented subspace, lifting, Köthe sequence space, short exact sequence.

${ }^{1}$ This paper was presented at the VI Polish-GDR Seminar on Banach space theory, Georgenthal, April 1984.

C1986 American Mathematical Society $0002-9939 / 86 \$ 1.00+\$ .25$ per page 
following additional assumptions on $Y$ and/or $Z$ : (1) $\operatorname{dim} Y=1, Z$ Fréchet [5, Theorem 10.2]; (2) $\operatorname{dim} Y=1$ [1, Theorem 5.5(c)]; (3) $Z$ has a weak topology [1, Proposition 4.3]; (4) $Y \simeq c_{0}$ or $l_{\infty}(A)$ [2, Theorem 2.1, Corollary 4.1]. After submitting this paper for publication, the author received the newly published paper [14] based on the homological methods which contains an independently obtained characterization of Fréchet $Y$ such that $Z \in S_{c}(Y)$ for every nuclear $Z$ [14, Theorems 5.2 and 1.8]; it contains a weaker version of our Theorem 3.3.

Theorem 3.7 asserts that $Z \in S_{c}(Y)$ for all nuclear Fréchet spaces $Y$ and Banach spaces $Z$. This result is due to V. P. Palamodov [9, Theorem 9.1], with a rather complicated "homological" proof. Our proof is more direct and based on Vogt's results [13, Theorem 1.5$]$, (compare $[16,17,7])$.

The author is very indebted to the referee for his remarks.

1. General results on the splitting of twisted sums. The nonlocally convex version of Theorem 1.1 is proved in [2, Theorem 1.2]; the proof works in the locally convex case as well.

THEOREM 1.1. For every (locally convex) twisted sum (*) of tws (lcs) $Y$ and $Z$ and every tvs (lcs) $Y_{0} \supset{ }^{i} Y$, there is a tvs (lcs) $X_{0}$, and a topological embedding $T$ : $X \hookrightarrow X_{0}$ such that the following diagram, with both rows being twisted sums, commutes:

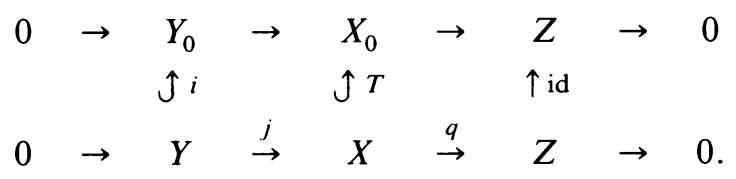

If $Z, Y_{0} \supset Y$ are tvs and $q: Y_{0} \rightarrow Y_{0} / Y$ is the natural quotient map, then a lifting of a continuous linear map $L_{0}: Z \rightarrow Y_{0} / Y$ is a continuous linear map $L: Z \rightarrow Y_{0}$, $q \circ L_{0}=L$. Now we establish some permanence properties of the classes $S(Y)$ and $S_{c}(Y)$.

Theorem 1.2. (a) If $Z \in S\left(Y_{i}\right)$ for tvs $Z, Y_{i}, i \in I$, then $Z \in S\left(\prod_{i \in I} Y_{i}\right)$.

(b) If $Z \supset Z_{0}$ and $Y$ are tvs and every continuous linear map $L_{0}: Z_{0} \rightarrow Y$ may be extended continuously to a linear map $L: Z \rightarrow Y$, then $Z / Z_{0} \in S(Y)$ whenever $Z \in S(Y)$.

(c) If $Y_{0} \supset Y$ and $Z$ are tvs, $Z \in S\left(Y_{0}\right)$, and every continuous linear map $L_{0}$ : $Z \rightarrow Y_{0} / Y$ may be lifted to $L: Z \rightarrow Y_{0}$, then $Z \in S(Y)$.

The assertions (a) and (c) hold if all spaces are lcs and classes $S(\cdot)$ are replaced by $S_{c}(\cdot)$.

Proof. (a) [2, Proposition 1.1] The locally convex version has the same proof.

(b) [1, Lemma 4.1].

(c) We prove only the general version since the proofs are similar. First, we extend (*), by 1.1 , to the twisted sum of $Y_{0}$ and $Z$. Thus we obtain the following 
commutative diagram, where rows are twisted sums, $p \circ j_{0}=\mathrm{id}, q_{0}: Y_{0} \rightarrow Y_{0} / Y$ and $q_{1}, p, j_{0}$, and $i$ are the natural quotient map, a projection, and embeddings, resp.:

$$
\begin{array}{ccccccccc}
0 & \rightarrow & Y_{0} & \stackrel{j_{0}}{\rightleftarrows} & Y_{0} \oplus Z & \stackrel{q_{1}}{\rightarrow} & Z & \rightarrow & 0 \\
& i \uparrow & & \uparrow T & & \uparrow \text { id } & & \\
0 & \rightarrow & Y & \stackrel{j}{\rightarrow} & X & \stackrel{q}{\rightarrow} & Z & \rightarrow & 0
\end{array}
$$

There is a continuous linear map $L_{0}: Z \rightarrow Y_{0} / Y$ such that $q_{0} \circ p \circ T=L_{0} \circ q$, because

$$
\begin{aligned}
\operatorname{ker}\left(q_{0} \circ p \circ T\right) & =T^{-1}\left(p^{-1}\left(p \circ j_{0} \circ i(Y)\right)\right) \supset T^{-1}(T \circ j(Y)) \\
& =j(Y)=\operatorname{ker} q .
\end{aligned}
$$

By the assumption, there exists a lifting $L_{1}: Z \rightarrow Y_{0}$ of $L_{0}, L_{0}=q_{0} \circ L_{1}$. For every $x \in X, L_{1} \circ q(x)-p \circ T(x) \in \operatorname{ker} q_{0}=i(Y)$. Hence for some $y \in Y$

$$
\left(L_{1} \circ q(x), q(x)\right)=\left(p \circ T(x+j(y)), q_{1} \circ T(x+j(y))\right)=T(x+j(y))
$$

because $p \circ T \circ j(y)=i(y)$. Thus the map $L_{2}: Z \rightarrow Y_{0} \times Z, L_{2}(z)=\left(L_{1}(z), z\right)$ fulfils $L_{2}(Z) \subset T(X)$. The map $s: Z \rightarrow X, s(z)=T^{-1}\left(L_{2}(z)\right)$ is the linear continuous section for $q$ we are looking for because

$$
q \circ T^{-1} \circ L_{2}(z)=q \circ T^{-1}\left(L_{1}(z), z\right)=q_{1}\left(L_{1}(z), z\right)=z .
$$

$\tilde{X}$ denotes the direct sum of the closure of $\{0\}$ in $X$ and the completion of the Hausdorff tvs associated with $X$. If $j: Y \rightarrow X$ is a continuous linear map, $\tilde{j}$ is its extension from $\tilde{Y}$ to $\tilde{X}$.

Lemma 1.3 [1, Corollary 2.1]. Let $Y$ be a semimetrizable tvs and let $Z$ be $a$ Hausdorff tvs. If $(*)$ is their twisted sum, then

$$
0 \rightarrow \tilde{Y} \stackrel{\tilde{j}}{\rightarrow} \tilde{X} \stackrel{\tilde{q}}{\rightarrow} \tilde{Z} \rightarrow 0
$$

is a twisted sum too.

THEOREM 1.4. The class $S_{c}(Y)$ is closed under formation of reduced projective limits (i.e., canonical projections on factors possess dense images) of Hausdorff lcs whenever $Y$ is a Banach space.

Proof. Let us consider the following twisted sum:

$$
0 \rightarrow(Y,\|\cdot\|) \stackrel{j}{\rightarrow}(X, \tau) \stackrel{q}{\rightarrow}(Z, \gamma) \rightarrow 0,
$$

where $(X, \tau)$ is an lcs and $(Z, \gamma)$ is a reduced projective limit of the family $\left(Z_{i}\right)_{i \in I}$ of Hausdorff lcs, $Z_{i} \in S_{c}(Y)$ for every $i \in I$. We choose an absolutely convex 0 -neighbourhood $U$ in $(X, \tau)$ such that $j^{-1}(U)$ is contained in the unit ball of $(Y,\|\cdot\|)$. Thus for some $i_{0} \in I, p_{i_{0}}(q(U))$ is a 0 -neighbourhood in $Z_{i_{0}}$, where $p_{i_{0}}$ : $Z \rightarrow Z_{i_{0}}$ is the canonical projection. If $\gamma_{1}$ is the topology induced on $Z$ by $p_{i_{0}}$, then $\left(Z / \operatorname{ker} \gamma_{1}, \gamma_{1} / \operatorname{ker} \gamma_{1}\right)$ is isomorphic to a dense subspace of $Z_{i_{0}}$. All sets of the form $(1 / n) U \cap q^{-1}(V)$, where $V$ is a $\gamma_{1}$-neighbourhood of zero, $n \in N$, are a base at zero 
of the linear topology $\tau_{1}$ on $X$ such that the following diagram forms a twisted sum:

$$
0 \rightarrow(Y,\|\cdot\|) \stackrel{j}{\rightarrow}\left(X, \tau_{1}\right) \stackrel{q}{\rightarrow}\left(Z, \gamma_{1}\right) \rightarrow 0 .
$$

Hence $\operatorname{ker} \gamma_{1}=q\left(\operatorname{ker} \tau_{1}\right)$, and we can find subspaces $X_{1} \subset X$ and $Z_{1}=q\left(X_{1}\right) \subset Z$, $j(Y) \subset X_{1}$, such that algebraically $X_{1}$, $\operatorname{ker} \tau_{1}$ and $Z_{1}$, ker $\gamma_{1}$ are pairs of complements. Obviously $\left(Z_{1}, \gamma_{1} \cap Z_{1}\right) \simeq\left(Z / \operatorname{ker} \gamma_{1}, \gamma_{1} / \operatorname{ker} \gamma_{1}\right)$ and, by 1.3 , we get the twisted sum

$$
0 \rightarrow(Y,\|\cdot\|) \stackrel{j}{\rightarrow}\left(X_{2}, \tau_{2}\right) \stackrel{q_{1}}{\rightarrow} Z_{i_{0}} \rightarrow 0,
$$

where $X_{2}=\tilde{q}^{-1}\left(Z_{i_{0}}\right) \cap \tilde{X}_{1}, q_{1}=\left.\tilde{q}\right|_{X_{2}}, \tau_{2}=\widetilde{\tau_{1} \cap X_{1}} \cap X_{2}$ and $Z_{i_{0}}$ is identified with a dense subspace of $\left(\tilde{Z}_{1}, \gamma_{1} \cap Z_{1}\right)$ containing $Z_{1}$. There is a continuous linear section $s_{1}$ for $q_{1}$ because $Z_{i_{0}} \in S_{c}(Y)$. Let $s_{2}$ be a linear section for $q, s_{2}\left(\operatorname{ker} \gamma_{1}\right)=\operatorname{ker} \tau_{1}$. Then

$$
s:\left(Z, \gamma_{1}\right) \rightarrow\left(X, \tau_{1}\right), \quad s=s_{2} \circ r+s_{1} \circ(\mathrm{id}-r),
$$

is a continuous linear section for $q$ whenever $r: Z \rightarrow \operatorname{ker} \gamma_{1}$ is a projection onto, $\operatorname{im}(\mathrm{id}-r)=\operatorname{ker} r=Z_{1} \subset Z_{i_{0}}$. Thus $j(Y)$ is complemented in $\left(X, \tau_{1}\right)$ and in $(X, \tau)$ because $\tau$ and $\tau_{1}$ coincide on $j(Y)$.

REMARK. Theorem 1.4 is valid for $S(Y)$, where $Y$ is locally bounded and complete [1, Theorem 4.3(a)].

An lcs $Z$ is a TSC-space [1] iff for every lcs $Y$ every twisted sum of $Y$ and $Z$ is locally convex.

TheOREM 1.5 [2, Theorem 2.1]. An lcs $Z$ is a TSC-space iff $Z \in S\left(l_{\infty}(A)\right)$ for every set $A$. For metrizable lcs $Z$, the classes of $\mathscr{K}$-spaces and TSC-spaces coincide.

2. Applications to Köthe sequence spaces. Now, we will derive some consequences of the above results for Köthe spaces, i.e. spaces of the form

$$
\Lambda(P)=\left\{x=\left(x_{n}\right) \in K^{N}:\|x\|_{a}=\sum_{n=1}^{\infty} a_{n}\left|x_{n}\right|<\infty \text { for every } a=\left(a_{n}\right) \in P\right\},
$$

with the topology generated by the family of seminorms $\|\cdot\|_{a}, a \in P . P$ is an arbitrary set of nonnegative sequences which fulfil conditions:

(i) for every $m \in N$ there is $\left(a_{n}\right) \in P$ such that $a_{m} \neq 0$,

(ii) for every $\left(a_{n}\right),\left(b_{n}\right) \in P$ there is $\left(c_{n}\right) \in P$ such that $c_{n}>\max \left(a_{n}, b_{n}\right), n \in N$.

LEMMA 2.1. Every locally convex twisted sum of an arbitrary Banach space $Y$ and the Banach space $l_{1}$ splits.

Proof. Every such twisted sum is complete by [12], and locally bounded by [4, Theorem 1.1], so Banach. It splits by the well-known lifting property of $l_{1}[3$, Theorem 6.9.5].

TheOREM 2.2 (COMPARE [15, Lemma 1.4, Remark (1), p. 6]). Every locally convex twisted sum of a Banach space $Y$ and a Köthe space $Z$ splits.

Proof. Obviously $Z$ is a reduced projective limit of spaces isomorphic to $l_{1}$. Thus 2.1 and 1.4 justify the theorem. 
COROllary 2.3. If $Z$ and $Z / Y$ are Köthe spaces for some infinite-dimensional Banach subspace $Y$, then $Y \simeq l_{1}$.

Proof. By 2.2, there is a continuous projection $P: Z \rightarrow Y$. Hence for some standard seminorm $\|\cdot\|_{a}$ on the Köthe space $Z, P:\left(Z,\|\cdot\|_{a}\right) \rightarrow Y$ is also a continuous projection. Finally, $Y \simeq l_{1}$, because $\left(\widetilde{Z,\|\cdot\|_{a}}\right) \simeq l_{1}$ and $Y$ is complemented (cf. [8, Theorem 2.a.3]).

COROllary 2.4. For a Banach space $Y, \Lambda(P) \in S(Y)$ iff for every $\left(a_{n}\right) \in P$ there are $\left(b_{n}\right) \in P$ and $t<\infty$ such that

$$
\text { (+) } \quad \sum_{n=1}^{\infty} \exp \left(-t b_{n} / a_{n}\right)<\infty \quad(0 / 0=\infty) \text {. }
$$

In particular, a Köthe space is a TSC-space iff it is a $\mathscr{K}$-space.

Proof. For metrizable $\Lambda(P)$ and $\operatorname{dim} Y=1$ this is shown in [5, Theorem 10.3]. The same arguments show that if $(+)$ fails, then a nonmetrizable $\Lambda(P)$ is not a $\mathscr{K}$-space. Hence there is a nonlocally convex twisted sum of $K(\operatorname{dim} K=1)$ and $\Lambda(P)$, extendable, by 1.1 , to a nonsplitting twisted sum of $Y$ and $\Lambda(P)$.

Conversely, if $(+)$ is valid, then $\Lambda(P)$ is a reduced projective limit of a family of metrizable spaces $\Lambda\left(P_{i}\right), i \in I$, each of them satisfying $(+)$. Thus $\Lambda\left(P_{i}\right)$ are $\mathscr{K}$-spaces, and by $1.5, \Lambda\left(P_{i}\right) \in S\left(l_{\infty}(A)\right)$ for every set $A$. The nonlocally convex version of 1.4 (see the remark after its proof) asserts that $\Lambda(P)$ belongs to $S\left(l_{\infty}(A)\right.$ ) for every set $A$. By $1.5, \Lambda(P)$ is a TSC-space. This completes the proof, by 2.2 .

3. Applications to nuclear spaces. The Köthe space $\Lambda(P), P=\left\{a^{k}=\left(n^{k}\right)\right.$ : $k \in \mathbf{N}\}$, is usually denoted by $s$.

THEOREM 3.1. Let $X$ be a nuclear lcs.

(a) [3, Theorem 21.7.1] $X$ is isomorphic to a subspace of $s^{I}$ for a suitable set I which may be chosen countable if $X$ is Fréchet.

(b) $[3$, Corollary $21.2 .5 ; 11]$ Let $Z$ be a separable infinite-dimensional Banach space. Then $X$ is isomorphic to a dense subspace of a reduced projective limit of a family of spaces $X_{i}$ such that $X_{i}$ is either finite-dimensional or isomorphic to $Z$.

(c) [1, Theorem 5.5(c) or 2, Corollary 4.1] X is a TSC-space.

The following Proposition 3.2 is surely known.

Proposition 3.2. Let $Z_{1} \subset Z$ be Banach spaces and let $Y_{1} \subset Y$ be nuclear spaces.

(a) Every continuous linear map $L: Y_{1} \rightarrow Z\left(L: Y \rightarrow Z / Z_{1}\right)$ may be extended (lifted) to a continuous linear map $L_{1}: Y \rightarrow Z$.

(b) If $Y$ is a Fréchet space, then every continuous linear map $L: Z \rightarrow Y / Y_{1}$ may be lifted to a map $L: Z \rightarrow Y$.

Proof. (a) is a straightforward application of the Hahn-Banach theorem for nuclear operators (cf. [10, III.7.2 and III.7.1]). To prove (b) it is enough to extend the adjoint map $L^{\prime}$ to all of $Y^{\prime}$ (the strong dual of a Fréchet nuclear space is nuclear [10, IV.9.6]; use (a)). Then the adjoint map of this extension restricted to $Z \subset Z^{\prime \prime}$ is the 
lifting we are looking for because any nuclear Fréchet space is reflexive (cf. [10, III.7.2, Corollary 2]).

Now, we have our first main theorem, with three different proofs. Another proof ("homological") for locally convex twisted sums was given independently by D. Vogt [14, Corollary 1.2, Theorem 1.8 or 15, Lemma 1.4 and Remark, p. 6].

THEOREM 3.3. Every twisted sum of a Banach space $Y$ and a nuclear space $Z$ splits.

Proof. I. By 3.1(b), $\tilde{Z}$ is a reduced projective limit of finite dimensional spaces and spaces isomorphic to $l_{1}$. Thus our result is an immediate consequence of $1.3,2.1$, 1.4 and $3.1(\mathrm{c})$.

II. Of course, $Y \subset l_{\infty}(A)=Y_{0}$ for a suitable set $A$. By 3.1(c) and 1.5, $Z \in$ $S\left(l_{\infty}(A)\right)$. Now, by $1.2(\mathrm{c})$ and $3.2($ a) we get $Z \in S(Y)$.

III (Proof for Fréchet $Z$ ). By 3.1(c) and 2.2, our theorem holds if $Z$ is a Köthe nuclear space. But Wagner [18] showed that every Fréchet nuclear space is isomorphic to a quotient of a Köthe nuclear space. The theorem is a consequence of 3.2(a) and 1.2(b).

REMARK. In proof I it is not enough to use existence of enough " $l_{p}$-seminorms" instead of 3.1 because we require the reduced (!) projective limit representation.

As a corollary we get (by 1.1, 1.2(a)) the following "representation theorem" for twisted sums (compare [1, Theorem 3.1], [2, Theorem 1.3] and the incorrect [7, Theorem 2.5]). A similar result holds in more generality.

THEOREM 3.4. Let $Y$ be an arbitrary lcs and let $Y_{0}$ be an arbitrary product of Banach spaces, $Y_{0} \supset Y$. Every twisted sum of $Y$ and a nuclear lcs $Z$ may be extended (as in 1.1) to a direct sum of $Y_{0}$ and $Z$.

Now, let us consider the pair of lcs $(Y, Z), Y$ Fréchet nuclear, $Z$ Banach. By the remarks in $[13$, p. 116], the following theorem is a particular case of [13, Theorem 1.5]. The corollary is implied by $1.2(\mathrm{a})$ and 1.5 .

THEOREM 3.5. Every Banach space belongs to $S_{c}(s)$.

COROLlary 3.6. For every set I the class $S_{c}\left(S^{I}\right)$ contains all Banach spaces and $S\left(s^{\prime}\right)$ contains all Banach $\mathscr{K}$-spaces.

Kalton [4, Theorem 2.6] proved that every superreflexive, in particular uniformly convex, Banach space is a $\mathscr{K}$-space. The first part of the following theorem is due to V. P. Palamodov [9, Theorem 9.1] who assumed only that $Z$ is "dual metric".

THEOREM 3.7. Every locally convex twisted sum of a nuclear Fréchet space $Y$ and a normed space $Z$ splits. Every twisted sum of $Y$ and $Z$ splits iff $Z$ is a $\mathscr{K}$-space, in particular if $Z$ is superreflexive.

Proof. By 1.3 , we can assume that $Z$ is complete. If $Z$ is not a $\mathscr{K}$-space, then there is a nonlocally convex twisted sum of $K$ and $Z$. By 1.1 , it may be extended to the nonlocally convex (i.e., nonsplitting) twisted sum of $Y$ and $Z$. 
Now, by 1.5 , it is enough to prove the first part of the theorem. By 3.6, $Z \in S_{c}\left(s^{N}\right)$, thus our theorem is an immediate consequence of $3.1(\mathrm{a}), 1.2(\mathrm{c})$ and 3.2(b).

\section{REFERENCES}

1. P. Domanski, On the splitting of twisted sums, and the three space problem for local convexity, Studia Math. 82 (1985), 49-83.

2. L L L L L Lal convexity of twisted sums, Suppl. Rend. Circ. Mat. Palermo Serie II, 5 (1984), Proc. XII Winter School on Abstract Analysis, Srni, 1984, pp. 13-31.

3. H. Jarchow, Locally convex spaces, Teubner, Stuttgart, 1981.

4. N. J. Kalton, Three space problem for locally bounded F-spaces, Compositio Math. 37 (1978), $243-276$.

5. Convexity, type and the three space problem, Studia Math. 69 (1981), 247-287.

6. N. J. Kalton and N. T. Peck, Quotients of $L_{p}(0,1)$ for $0 \leqslant p<1$, Studia Math. 64 (1979), 65-75.

7. T. Ketonen and K. Nyberg, Twisted sums of nuclear Frechet spaces, Ann. Acad. Sci. Fenn. Ser. AI Math. 7 (1982), 323-335.

8. J. Lindenstrauss and L. Tzafriri, Classical Banach spaces, Vol. I, Springer-Verlag, Berlin, 1977.

9. V. P. Palamodov, Homological methods in the theory of locally convex spaces, Uspekhi Mat. Nauk 26 (1971), 3-66. (Russian)

10. H. H. Schaefer, Topological vector spaces, Springer-Verlag, New York, 1971.

11. M. Valdivia, Nuclearity and Banach spaces, Proc. Edinburgh Math. Soc. (2) 20 (1977), 205-209.

12. N. Ya. Vilenkin, On the theory of weakly separable groups, Mat. Sb. 22 (1948), 135-177. (Russian)

13. D. Vogt, Charakterisierung der Unterräume von s, Math. Z. 155 (1977), 109-117.

14. Some results on continuous linear maps between Fréchet spaces, Functional Analysis: Surveys and Recent Results. III, North-Holland Math. Studies, Vol. 90, North-Holland, Amsterdam, 1984, pp. $349-381$.

15. On the functors $\operatorname{Ext}^{1}(E, F)$ for Fréchet spaces, preprint.

16. D. Vogt and M. J. Wagner, Charakterisierung der Unterräume und Quotientenräume der nuklearen stabilen Potenzreihenräume von unendlichem Typ, Studia Math. 70 (1981), 63-80.

17. Charakterisierung der Quotientenräume von $s$ und eine Vermutung von Martineau, Studia Math. 67 (1980), 225-240.

18. M. J. Wagner, Jeder nukleare ( $F)$-Raum is Quotient eines nuklearen Köthe-Raumes, Arch. Math. 42 (1983), 169-175.

Institute of Mathematics, A. Mickiewicz University, Matejki 48 / 49, 60-769 Poznań, Poland 